Draft VERSion FEBRUARY 13, 2020

Preprint typeset using LATEX style AASTeX6 v. 1.0

\title{
DATA-DRIVEN MHD SIMULATION OF THE FORMATION AND INITIATION OF A LARGE-SCALE PRE-FLARE MAGNETIC FLUX ROPE IN SOLAR ACTIVE REGION 12371
}

\author{
Wen He ${ }^{1,3}$, Chaowei Jiang ${ }^{* 1}$ Peng Zou ${ }^{1}$, Aiying Duan ${ }^{2}$, Xueshang Feng ${ }^{1}$, Pingbing Zuo ${ }^{1}$, Yi Wang ${ }^{1}$ \\ ${ }^{1}$ Institute of Space Science and Applied Technology, Harbin Institute of Technology, Shenzhen 518055, China; chaowei@hit.edu.cn \\ ${ }^{2}$ School of Atmospheric Sciences, Sun Yat-sen University, Zhuhai, Guangdong 519082, China \\ ${ }^{3}$ Center for Space Plasma and Aeronomic Research, The University of Alabama in Huntsville, Huntsville, AL 35899, USA
}

\begin{abstract}
Solar eruptions are the most powerful drivers of space weather. To understand their cause and nature, it is crucial to know how the coronal magnetic field evolves before eruption. Here we study the formation process of a relatively large-scale magnetic flux rope (MFR) in active region NOAA 12371 that erupts with a major flare and coronal mass ejection on 2015 June 21. A data-driven numerical magnetohydrodynamic model is employed to simulate three-dimensional coronal magnetic field evolution of one-day duration before the eruption. Comparison between the observed features and our modeled magnetic field discloses how the pre-eruption MFR forms. Initially, the magnetic field lines were weakly twisted as being simple sheared arcades. Then a long MFR was formed along the polarity inversion line due to the complex photospheric motion, which is mainly shearing rather than twisting. The presence of the MFR is evidenced by a coherent set of magnetic field lines with twist number above unity. Below the MFR a current sheet is shown in the model, suggesting that tether-cutting reconnection plays a key role in the MFR formation. The MFR's flux grows as more and more field lines are twisted due to continuous injection of magnetic helicity by the photospheric motions. Meanwhile, the height of the MFR's axis increases monotonely from its formation. By an analysis of the decay index of its overlying field, we suggest that it is because the MFR runs into the torus instability regime and becomes unstable that finally triggers the eruption.
\end{abstract}

Keywords: Sun: corona; Sun: magnetic fields; Sun: coronal mass ejections (CMEs); Sun: flares; magnetohydrodynamics (MHD); methods: numerical

\section{INTRODUCTION}

Large-scale eruptions occurring in the solar atmosphere can release a vast amount of energy up to $10^{32} \mathrm{erg}$ in tens of minutes and may severely affect the space environment around the Earth. Such phenomena including flares, filament eruptions and coronal mass ejections (CMEs) are driven commonly by the Sun's magnetic field evolution. In particular, the magnetic field plays a dominant role in the solar corona because the plasma $\beta$, i.e., ratio of gas pressure to magnetic pressure, is often very small. The coronal magnetic field can be strongly stressed by photospheric flux emergences and motions, and excess or free magnetic energy is accumulated until a catastrophic release occurs, which powers solar eruption events (Aschwanden 2004). During solar eruptions, magnetic reconnection is thought to be the key mechanism that converts magnetic free energy to radiation and energetic particle acceleration (Priest \& Forbes 2002). Meanwhile, it cuts parts of the connection of the magnetic flux with the Sun and allows a huge amount of magnetized plasma to be ejected into inter- planetary space as coronal mass ejections. Since variation of magnetic field topology has a close relationship with magnetic reconnection, it is essential to understand the evolution of magnetic configuration in the corona to figure out the nature and cause of solar eruptions.

Direct and accurate measurement of the magnetic field is less accessible in the chromosphere and corona than in the photosphere due to the low density and high temperature, which gives rise to many theoretical models being proposed. For example, the standard CME/flare model is frequently mentioned (Carmichael 1964; Sturrock 1966; Hirayama 1974; Kopp \& Pneuman 1976). It introduces a conceptual scenario that a magnetic flux rope (MFR) in the corona is ejected into interplanetary space. An MFR is a bundle of twisted magnetic field lines lying above the polarity inversion line (PIL) of photospheric magnetic field, with two legs anchored at the photosphere and, some parts of field lines of the MFR may be manifested as different observable features such as filaments, Sigmoids or hot channels (Cheng et al. 2013, 2014). With the rising of the MFR, its overlying 
field is strongly stretched and squeezed below the MFR, where an electric current sheet is formed and reconnection sets in. Then, part of the magnetic energy released in the reconnection tracks the newly reconnected field lines to the chromosphere and results in two parallel flare ribbons at the footpoints of these field lines (Benz 2017).

However, these theoretical models are idealized or hypothetical simplification of the realistic solar eruptions that is much more complex and elusive than what the standard model shows (e.g. Jiang et al. 2018a). For example, the nature of the pre-eruption configuration is still elusive. There are intensive debates on whether MFR exists before flare or forms during flare (Forbes 2000; Moore et al. 2001; Chen 2011). A conclusive answer to this question would provide a specific guidance to our understanding of solar eruptions. Although a lot of evidence is found for that MFR could exist prior to eruptions (Cheng et al. 2017), there is still no consensus on how and where an MFR can form. One supposition is that the MFR can bodily emerge from below the photosphere by buoyancy (Fan 2001; Martínez-Sykora et al. 2008; Magara 2004; Archontis et al. 2009). The other supposes that the MFR can be built up directly in the corona via magnetic reconnection prior to the eruption (van Ballegooijen \& Martens 1989; Aulanier et al. 2010; Amari et al. 2003). Moreover, it was claimed that some MFRs might be formed during eruption (Ouyang et al. 2017).

Despite of the fact that routine observations of the photospheric magnetograms have been made in the past decades, reliable measurement of the full 3D magnetic field in the solar atmosphere is still unavailable. $\mathrm{Nu}$ merical simulations based on the magnetohydrodynamics (MHD) model prove to be a powerful tool to reproduce the time-dependent, nonlinear evolution process of the 3D magnetic configuration and investigate the dynamic evolution of solar eruptions. For instance, the formation of an MFR directly in the corona and its eruption have been extensively studied (Aulanier et al. 2010, 2012). However, such idealized configuration of MFR might not be able to characterize the realistic case in the solar corona. In order to account for the complexity and evolution of the magnetic configuration in the real scene, a detailed and accurate description of evolving magnetic field is required. Realistic simulations of solar eruptions driven directly or constrained by photospheric magnetograms provide an important way to this end (e.g., Wu et al. 2006; Cheung \& DeRosa 2012; Jiang \& Feng 2013). Very recently, such data-driven numerical simulations are becoming an more and more active research field for solar eruptions. For instance, Jiang et al. (2016) developed a data-driven MHD model that self-consistently follows the time-sequence of ob- servations. Nayak et al. (2019) studied the magnetic reconnection process of a blowout jet and a flare with a data-constrained MHD simulation. Cheung et al. (2019) presented a comprehensive radiative MHD simulation of a solar flare to capture the process from emergence to eruption. Time-dependent photospheric electric field and plasma flow data were used by Hayashi et al. (2019) to conduct a data-driven MHD simulation for solar active region. Pomoell et al. (2019) analyzed the coronal response to the driving electric fields as boundary conditions of data-driven magnetofrictional simulation for the evolution of coronal magnetic field. Guo et al. (2019) recently developed a data-driven MHD model using the zero- $\beta$ approximation and successfully simulated an MFR eruption in consistent with multi-wavelength observations.

In this paper, we conduct a data-driven MHD modelling study for the formation process of an pre-eruption MFR, which helps to identify the mechanisms of its build-up process and initiation. The eruption event occurred in NOAA AR 12371 on 2015 June 21. Our datadriven MHD simulation reproduces the dynamic evolution of the 3D magnetic field covering one day before the flare onset time. The simulation clearly demonstrates the creation of a large-scale pre-flare MFR in the corona and its evolution until before the eruption. By comparison with observations and previous studies (Vemareddy 2017; Vemareddy \& Demóulin 2018), we found that this MFR is consistent with a long hot channel and filament observed by SDO/AIA. We further performed a detailed analysis of the building-up process, magnetic energy evolution and triggering mechanism of the MFR. The remainder of this paper is organized as follows. Data and method are presented in Section 2, then we analyze the evolution of magnetic configuration in Section 3 by observations and simulation results, and we conclude in Section 4.

\section{DATA AND METHOD}

\subsection{Event and Data}

AR 12371 owned a complex magnetic field configuration and launched four successive fast CMEs during its disk transit from 2015 June 18 to 25. These CMEs were associated with long-duration M-class flares on June 18, 21, 22, and 25, respectively. On June 21, a halo CME left the Sun when the AR was near the disk center (N12, E16) and generated a strong geomagnetic storm $\left(D_{s t}\right.$ index was -204 nT) on June 22. The CME was associated with an M2.6 flare, which started at around 1:00 UT on June 21. A recent study of this AR by Vemareddy \& Demóulin (2018) presented an analysis of the 3D magnetic field extrapolation with NLFFF model and studied these CMEs in relation to the coronal magnetic 


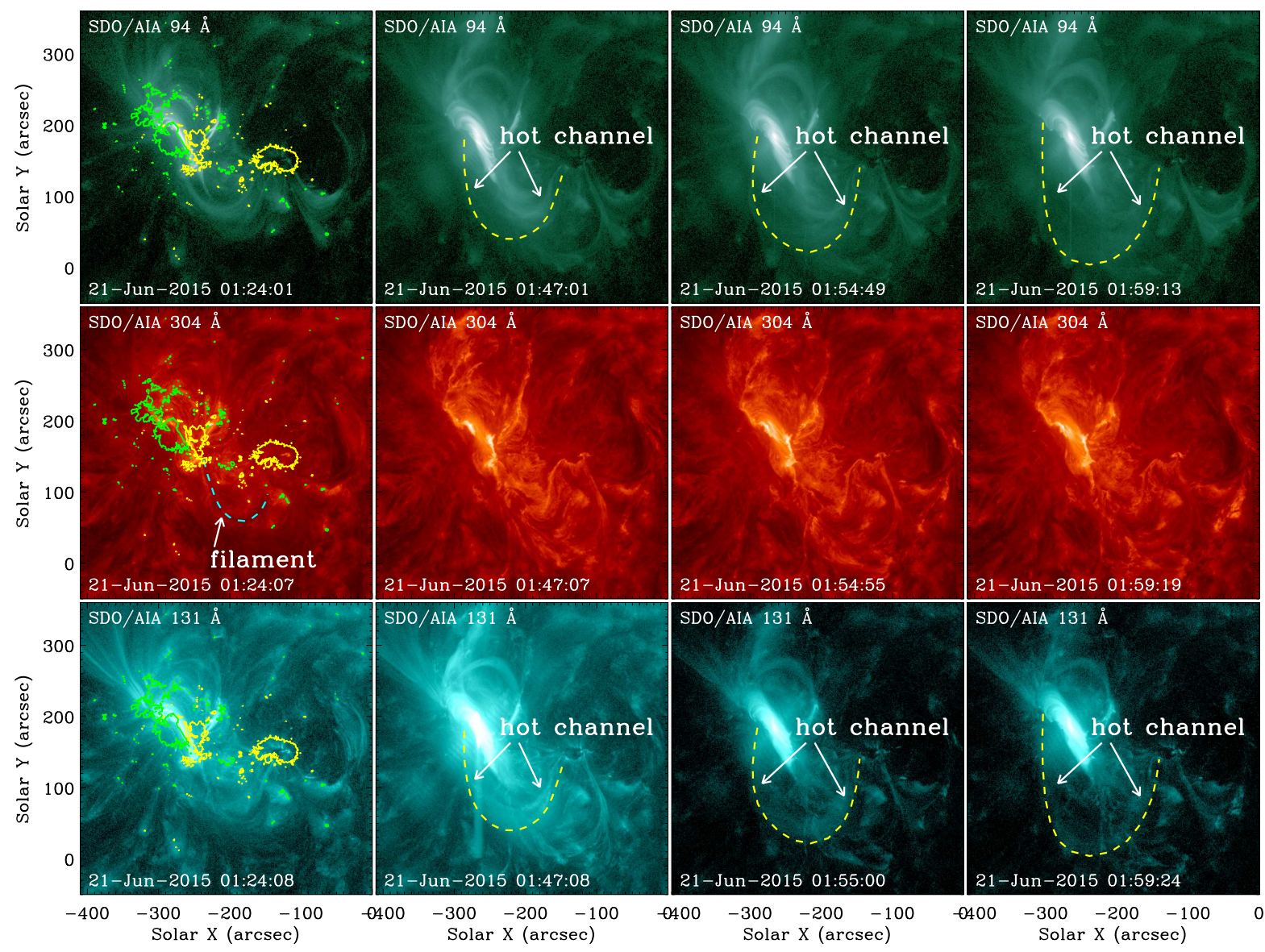

Figure 1. SDO/AIA observations of evolution in AR 12371 immediately before and during the eruption on early 2015 June 21. Each panel has the same field of view. From top to bottom are respectively $94 \AA, 304 \AA$ and $131 \AA$. The hot channel and filament are marked by the arrows. In the first column, the contours of line-of-sight magnetic field are shown with green for $500 \mathrm{G}$ and yellow for $-500 \mathrm{G}$.

evolution. We pay attention to their dynamic evolution specifically to disclose the underlying physics of this complex region. We utilized the EUV imaging data from the Atmospheric Imaging Assembly (AIA, Lemen et al. 2012) onboard the Solar Dynamic Observatory (SDO) to judge the temporal evolution of the AR at first. The $S D O /$ AIA provides full-disk coronal images in $7 \mathrm{EUV}$ filtergrams with pixel size of 0.6 arcsec and a cadence of $12 \mathrm{~s}$. Observations of the photospheric magnetic field were taken from $S D O /$ Helioseismeic and Magnetic Imager (HMI; Schou et al. 2012). Specifically, we choose the data product of the Space-weather HMI Active Region Patch (SHARPs, Bobra et al. 2014) as input to our model for driving the evolution of the coronal magnetic field.

\subsection{Data-driven MHD model}

We employed the data-driven active-region evolution MHD (DARE-MHD) model (Jiang et al. 2016) to simulate the coronal magnetic field evolution in response to the evolution of the photospheric magnetogram. In the DARE-MHD model, we solve the full set of 3D, time-dependent MHD equations with the magnetic field on the bottom boundary continuously provided by the vector magnetogram from $S D O / \mathrm{HMI}$. The initial condition consists of an extrapolated NLFFF data (descried in the next section) and a simple atmospheric model. The plasma is initialized in a hydrostatic, isothermal state with $T=10^{6} \mathrm{~K}$ in solar corona. To imitate the coronal low- $\beta$ and high tenuous conditions, the plasma density is configured to make the plasma $\beta$ less than 0.1 in most of the computational volume. At the bottom boundary, the plasma density and temperature is fixed, while the velocity is also inputted from observation-derived data using the DAVE4VM method (Schuck 2008). We use a non-uniform grid with adaptive resolution based on the spatial distributions of the magnetic field and current density, which is designed to save computational resources without losing numerical accuracy (Jiang et al. 2017). The smallest grid is $\Delta x=\Delta y=\Delta z=0.72 \mathrm{Mm}$. More details of the MHD simulation model can be found 
in Jiang et al. (2016, 2018b).

\subsection{NLFFF Extrapolation Model}

For a DARE-MHD simulation, an initial coronal magnetic field is needed. Here, the coronal magnetic field is extrapolated by the CESE-NLFFF code developed by Jiang et al. (2013). This model is based on an MHD-relaxation method which seeks an approximately force-free equilibrium. It solves a set of modified zero$\beta$ MHD equations with a friction force using an advanced conservation-element/solution-element (CESE) space-time scheme on a non-uniform grid with parallel computing (Jiang et al. 2010). The code also utilizes adaptive mesh refinement and a multi-grid algorithm to optimize the relaxation process. This model has been tested by different benchmarks including a series of analytic force-free solutions (Low \& Lou 1990) and MFR models (Titov \& Démoulin 1999). The results of extrapolation reproduced from $S D O / \mathrm{HMI}$ are in good agreement with corresponding observable features like filaments, coronal loops, and sigmoids (Jiang \& Feng 2013, 2014).

\subsection{Magnetic Field Analysis Tools}

The magnetic field data from our simulation is examined in several aspects including calculation of magnetic twist number $T_{w}$ for defining the MFR, decay index $n$ of the strapping field that confines the MFR, as well as magnetic squashing degree $Q$ which can used to locate critical thin layers where magnetic reconnection might take place. The magnetic twist number $T_{w}$ is defined by (Berger \& Prior 2006)

$$
T_{w}=\int_{L} \frac{(\nabla \times \mathbf{B}) \cdot \mathbf{B}}{4 \pi B^{2}} d l,
$$

where $L$ is along the magnetic field lines starting from one footpoint to the other on the bottom boundary. $T_{w}$ measures the number of winding turns between two infinitesimally close field lines (Liu et al. 2016), and clearly $T_{w}$ is a global parameter for any given field line. Here we compute $T_{w}$ for the whole $3 \mathrm{D}$ volume and then the MFR can be identified by coherent group of field lines with $T_{w} \geq 1$ (or $T_{w} \leq-1$ ). Thus by showing a isosurface of $\left|T_{w}\right|=1$ we can easily find the MFR in the full 3D volume (e.g., Duan et al. 2019). The decay index $n$ is calculated, which is defined by

$$
n=-\frac{\partial(\log B)}{\partial(\log h)} \text {. }
$$

It quantifies the spatial decaying speed of the strapping field strength $B$ with distance $h$ from the bottom surface. Here the strapping field is approximated by the potential field model extrapolated from the $B_{z}$ component of the photospheric magnetogram, and particularly, only the horizontal component $B_{\mathrm{h}}$ of the potential field is used as being the strapping field $B$. It would be more accurate if use only one component of $B_{\mathrm{h}}$ perpendicular to the PIL as the strapping field, because the MFR's axis is roughly parallel to the PIL. But for a potential field, its horizontal field is nearly perpendicular to the PIL. So computing decay index using total $B_{\mathrm{h}}$ should be close to that using only the perpendicular component. According to previous works, the torus instability of the constrained MFR will be triggered when it enters a domain with $n \gtrsim 1.5$ (Bateman 1978; Kliem \& Török 2006). We also derive the magnetic squashing degree ( $Q$ factor) based on the mapping of two footpoints for a field line (Démoulin 2006). This parameter can quantify the change of the field line linkage and locate prominent magnetic separatrix and thin layers, known as quasi-separatrix layers (QSL), where magnetic fieldline mapping changes abruptly and 3D magnetic reconnection is likely to occur (e.g. Priest \& Démoulin 1995; Aulanier et al. 2010; Démoulin 2006). Additionally, we use the distribution of ratio of current density to magnetic strength, $J / B$, to locate thin current layer in the simulation data. It has been shown that $J / B$ is a better indicator that can highlight current sheet-like distribution than the $J$ itself (Gibson \& Fan 2006; Fan \& Gibson 2007; Jiang et al. 2016).

\section{RESULTS}

\subsection{Basic configuration of the pre-flare corona}

Firstly we show the observed erupting structure of the flare. In Figure 1, simultaneous observations of SDO/AIA in different wavelengths, including 94, 304 and, $131 \AA$, present the evolution immediately before and during the eruption. From these observations, it can be seen that there is a large-scale, although rather faint, hot channel erupting toward the southern direction during the flare. The hot channel, as marked by the arrows in the figure, connects the east sunspot with the one in the southwest and forms an inverse J shape with its northeast part slightly hooked. Also there is an erupting filament of similar shape as shown in the images of AIA $304 \AA$. The presence of such hot channel as well as filament are often deemed to be manifestation of a corresponding MFR (Cheng et al. 2012; Zhang et al. 2012). More details of observation for this flare and eruption can be found in Vemareddy (2017) and Vemareddy \& Demóulin (2018).

Figure 2 compares a sequence of the photospheric magnetic configurations around the flare source region with the corresponding EUV observations. We also plot sampled magnetic field lines derived from the DAREMHD model and attached to the photosphere. From the photospheric magnetogram, we notice that this AR 

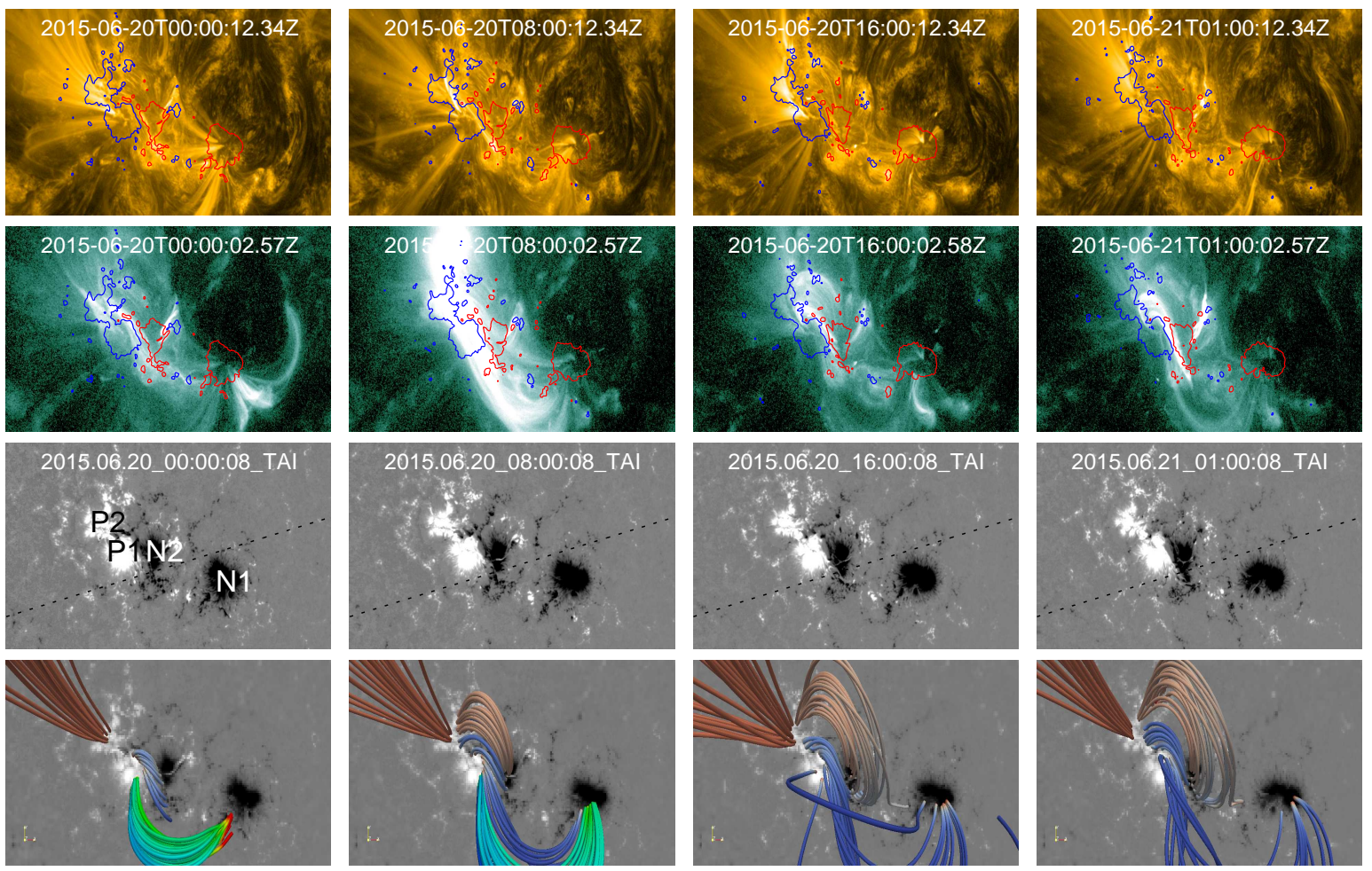

Figure 2. Comparison between the observed coronal loops of the AR and the modeled magnetic configuration. The first and second rows show EUV observations of AIA $171 \AA$ and $94 \AA$, respectively. Contour lines for $B_{z}=500 \mathrm{G}$ (blue) and $-500 \mathrm{G}$ (red) are overlaid. The third rows show the development of photospheric magnetic field observed by SDO/HMI. The last rows show sampled 3D magnetic field lines at corresponding time overlaid on the background magnetogram. The field of view of AIA observations are coaligned with HMI observations.

consists of a negative unipolar spot in the west and a bipolar spot group in the vicinity. Here the major magnetic polarities in the photosphere are marked as P1, P2, N1 and N2. A set of twisted field lines take shape of sigmoid structure lying along the PIL, which is in accordance with EUV observations and in turn suggests the existence of the MFR again, which is analyzed in details below. It's also worth mentioning that several field lines connect the positive region in the east to the negative region in the west, which matches well with the structure of coronal loops in $94 \AA$ images.

\subsection{Formation of a long flux rope}

The DARE-MHD simulation provides an important insight into the formation process of the long MFR. The initial time of the simulation is 00:00 UT on June 20. Time-dependent evolution of the magnetic structure in five time snapshots is shown from both top view and side view in Figure 3. Here we use isosurfaces with twist number $T_{w}=-1$ to show the position of the MFR, which is defined by a bundle of coherent twisted magnetic field lines with twist number above one turn. At the beginning, there is no MFR seen above the photosphere as the twist number $T_{w}$ is generally lower than one, thus, only sheared arcades around the PIL. Along with the dynamic evolution of the magnetic configura- tion, an MFR was gradually generated in the corona. Our simulation of the long MFR agrees well with the observation of EUV hot loops at 01:47 presented in Figure 1 . The magnetic twist number $\left(T_{w}<0\right)$ is significantly enhanced along two sides of the main PIL. As shown in the second and third column of Figure 3, overall the MFR continues to expand upward and extend its legs to two polarities far apart (N1 and P1 labelled in Figure 2). During the formation process of the MFR, there is hardly any emerging flux in this region observed from the magnetogram, and actually the total unsigned flux decreases in the duration (e.g., see Vemareddy 2017). So the long curved MFR can only be built up in the corona driven by the bottom surface motion rather than direct emergence. We suppose that the magnetic field lines around the MFR may intertwine and reconnect with each other through tether-cutting reconnection (Titov \& Démoulin 1999) after expansion and turn out to be a long MFR.

Further study on the evolution process of the MFR is revealed in Figure 4 in a vertical cross section which is perpendicular to the photosphere and along the dash lines as marked in Figure 2. We also plot the distribution of the $J / B$ (current density normalized by magnetic field strength) in the first column of Figure 4 for a better 

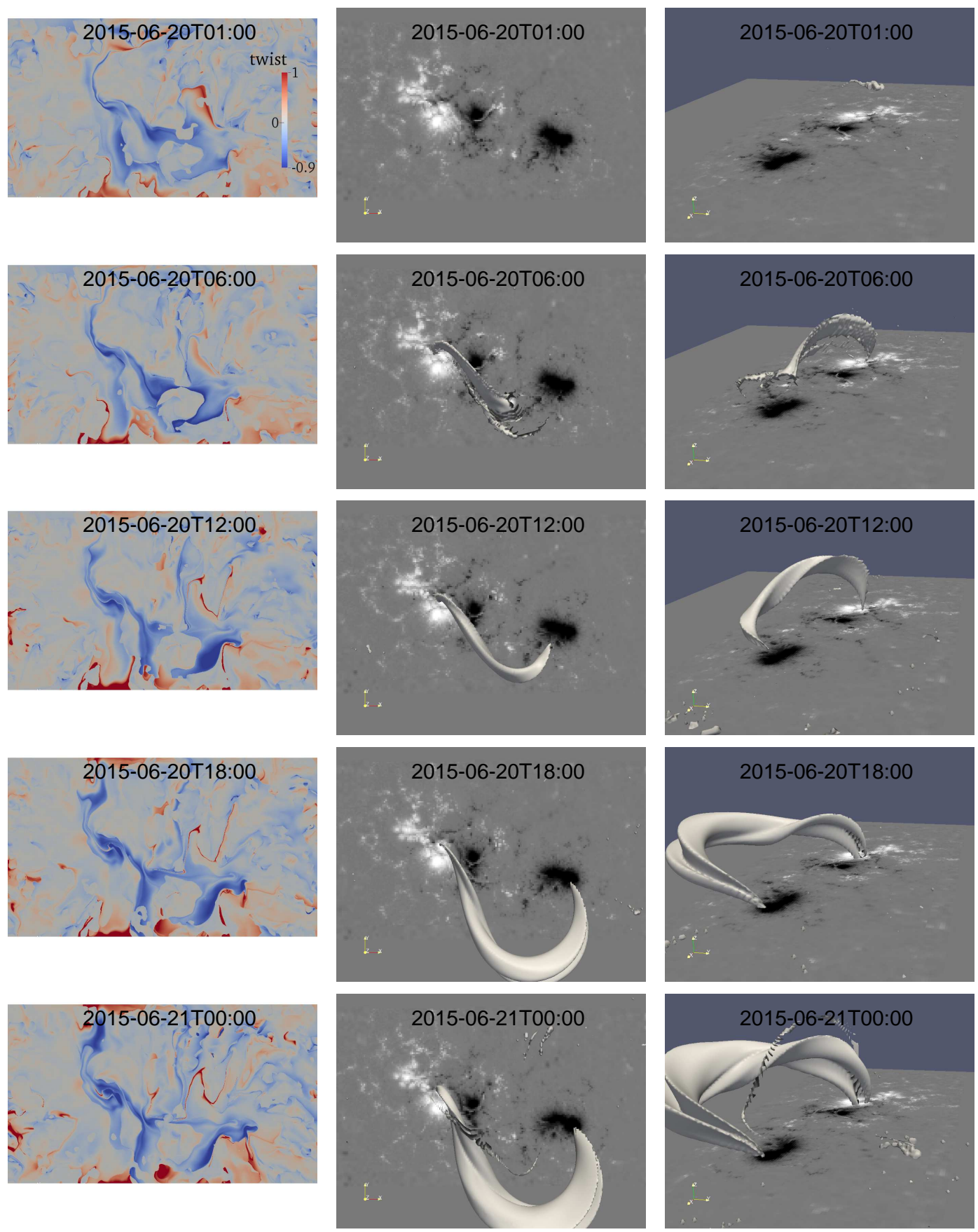

Figure 3. Evolution process of the MFR from a series of simulation time and different viewpoints. First column: distribution of $T_{w}$ on the photosphere. Second column: 3D structure of the MFR as shown by the isosurface of $T_{w}=-1$ overlaid on the background of photospheric $B_{z}$ map. Note that the map of magnetic twist number $T_{w}$ in the first panel has the same field of view of the photospheric magnetogram. The third column shows a side view of the same structure in the second column.

analysis of the current density. From the $Q$-factor maps in the second column, one can see that a QSL (with high $Q$ value) first forms above the PIL and then the MFR comes into being. With the generation of MFR, the QSL is further enhanced below the rope. This clearly suggests that the formation of the rope resulted from the reconnection in the QSL through a tether-cutting reconnection in the corona, rather than by a flux cancellation process where reconnection occurs in the photosphere. Indeed, along the QSL there forms a thin layer of strong current density, i.e., a current sheet that is associated with the reconnection, as can be seen in the first column of Figure 4. Matching current concentrations with the location of main QSLs, an MFR can be separated from its surroundings (Aulanier et al. 2010; Savcheva et al. 2012; Savcheva et al. 2012). It is worth noting that the 

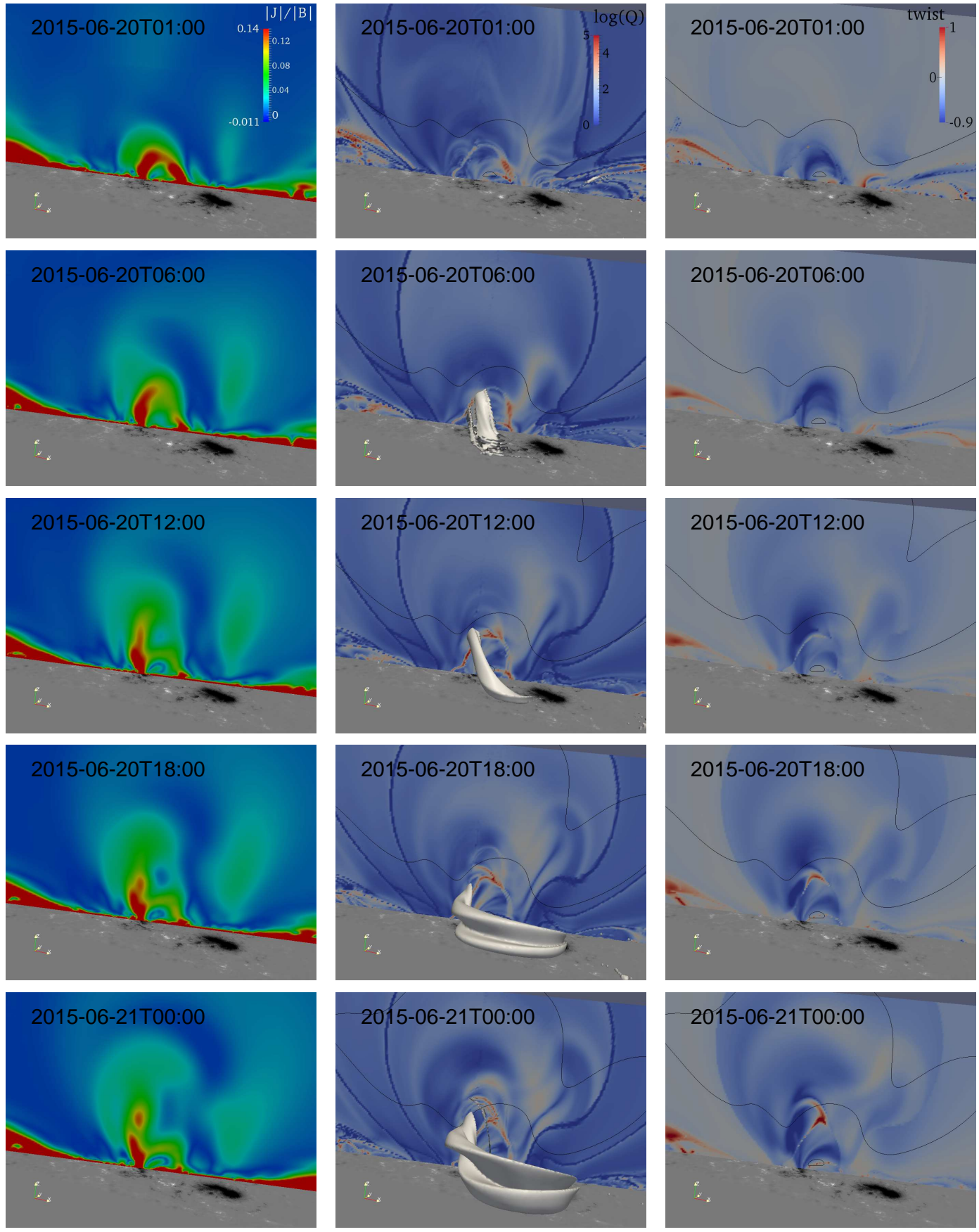

Figure 4. Evolution of magnetic structures in a vertical cross section that is along the line marked in Figure 2. All panels have the same viewing angle with photospheric $B_{z}$ map in the bottom. First column: distribution of the current density $J$ (normalized by magnetic field strength $B$ ) at different time. Second column: distribution of magnetic squashing degree $Q$. The narrow layers with high $Q$ (in red colors) are locations for magnetic topology separatrices and QSLs where magnetic field line mapping can change quickly due to reconnection. Third column: map of twists number $T_{w}$. The black contour lines of decay index $n=1.5$ are plotted in the second and third columns. 


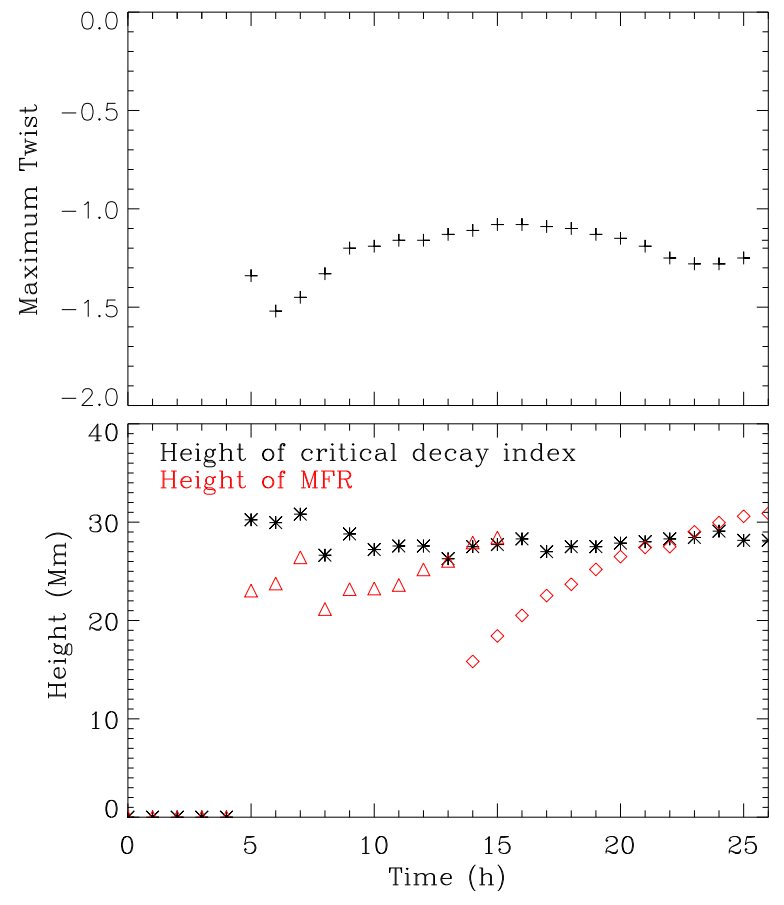

Figure 5. Evolution of the magnetic twist number and the height of the MFR as calculated from the vertical slice shown in Figure 4. Top panel: the maximum of the twist number in the rope, i.e., the twist number of the rope's axis. Bottom panel: the height of the apex of the rope axis, and the height with critical decay index equals 1.5. Note that at the time of $t=13$ the flux rope is split into two parts, and the triangles denotes the height of the upper one while the diamonds denotes the height for the lower one.

formation of the MFR can also be clearly seen from the evolution of the current density. For example, in the last panel of the first column of Figure 4, the sites of strong currents break into two parts while one part gradually rises up as a coherent circle, which corresponds to the MFR.

We further calculate the decay index $n$ in the vertical slice. At the early phase after the MFR formed, it lies relatively low and is far below the critical height with decay index of $n=1.5$. Then the MFR's axis is continuously lifted up in the corona. As shown in Figure 5, the maximum magnetic twist number in the MFR, with the value approximately at $-1 \sim-1.5$, does not change significantly during the evolution, suggesting that kink instability cannot be triggered. On the other hand, the height of the rope axis increases, and near the end of our simulation, for instance, at $t=24 \mathrm{~h}$, we notice that the major part of the MFR (the flux with $T_{w}<-1$ ) reaches a region with $n>1.5$. According to theoretical studies (Kliem \& Török 2006) and results of MHD simulations (Fan \& Gibson 2007; Aulanier et al. 2010), the torus instability (TI, Kliem \& Török 2006; Myers et al. 2015) occurs when the apex of the rope enters a region where decay index $n$ is larger than a threshold of $\sim 1.5$. The

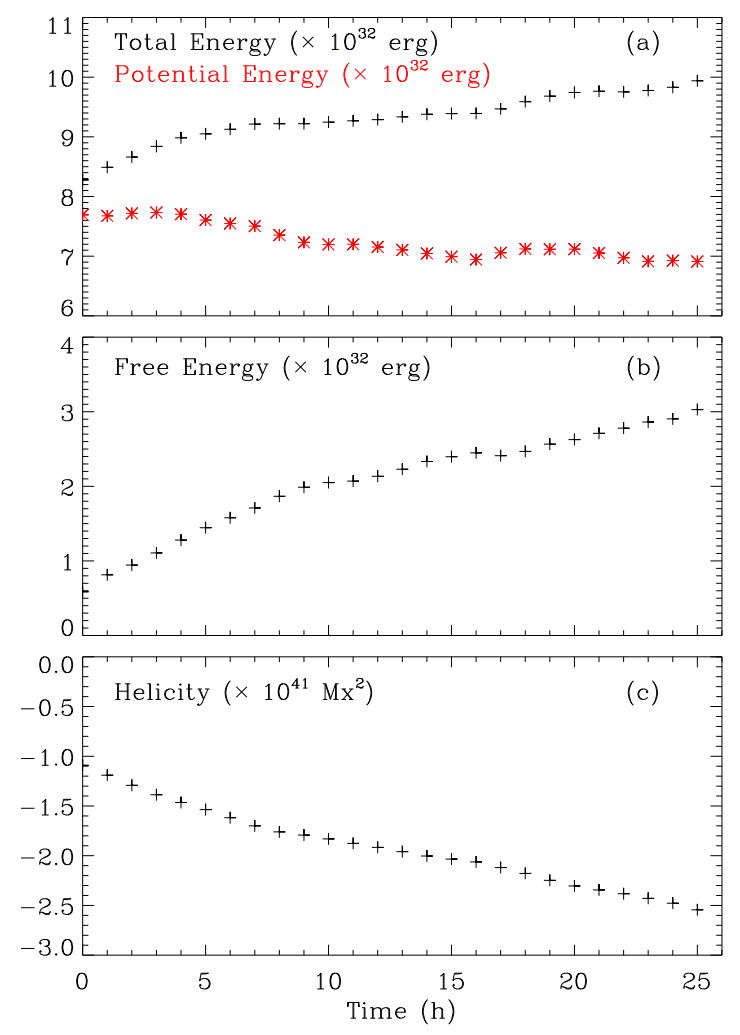

Figure 6. Evolution of magnetic energy (black, derived from the MHD model) and potential energy (red, derived from the potential field model), magnetic free energy and relative helicity from the data-driven MHD simulation.

TI is a kind of driver of MFR eruption, which is a result of the loss of equilibrium between the "hoop force" of the rope itself and the "strapping force" of the ambient field in idealized model. So in this event, the MFR has already reached an unstable region where the TI has a great potential to drive the eruption. However, a conclusion cannot be drawn directly since the TI theory is derived from idealized MFR configurations and realistic coronal magnetic field is much more complex. An interesting fact is found that the MFR is split into two parts during its evolution. As can be seen in the bottom panel of Figure 5, the upper part first reaches the critical height of TI and disappears at time of $t=14 \mathrm{~h}$. This splitting of the MFR might trigger a small flare and eruption. Such phenomena, however, are difficult to analyze in details with the rather low cadence and low resolution currently used in the model. There might be a possibility that the splitting results from the magnetic island generated in the reconnection, as a recent observation study shows (Gou et al. 2019), but still, to capture correctly the plasmoid in reconnection region requires very high resolution (such that the aspect ratio of the current sheet can be very large) which is out of the scope of the current paper. 


\subsection{Energies and magnetic helicity evolution}

To further study the global quantities of the magnetic field evolution, we calculate the magnetic energies and helicity. For instance, the free magnetic energy $\left(E_{\text {free }}\right)$ refers to the part of magnetic energy that can be released during eruptions. It can be derived by subtracting the potential energy $\left(E_{\text {pot }}\right)$ from the total magnetic energy $E_{\text {tot }}$,

$$
\begin{aligned}
E_{\text {free }} & =E_{\text {total }}-E_{\text {pot }} \\
& =\int_{V} \frac{B_{\text {tot }}^{2}}{8 \pi} d V-\int_{V} \frac{B_{\mathrm{pot}}^{2}}{8 \pi} d V
\end{aligned}
$$

where $V$ denotes the full computational volume of the simulation (note that here we used the CGS units). The evolution of total energy and free energy from $t=0$ to $t=26$ ( $1 \mathrm{hr}$ interval) of the MHD simulation system are plotted in Figure 6. As can be seen, the total magnetic energy keeps increasing while the potential field energy decreases. As a result, the free energy keeps increasing, which is consistent with the increasing of electric current in the corona. For the one day evolution, the amount of $E_{\text {free }}$ is accumulated to $\sim 2 \times 10^{32} \mathrm{erg}$. Apart from the free energy, the relative magnetic helicity is also a crucial indicator of the non-potential nature of the magnetic field (Berger \& Field 1984), especially for the existence of MFR. In a closed volume $V$, the relative magnetic helicity $H$ of a magnetic field $\mathbf{B}$ is defined as (Berger \& Field 1984; Finn 1984),

$$
H=\int_{V}\left(\mathbf{A}+\mathbf{A}_{\mathrm{p}}\right) \cdot\left(\mathbf{B}-\mathbf{B}_{\mathrm{p}}\right) d V
$$

where $\mathbf{B}_{\mathrm{p}}$ is the potential field with same magnetic flux distribution of $\mathbf{B}$ on the surface of the volume, and $\mathbf{A}$, $\mathbf{A}_{\mathrm{p}}$ are corresponding vector potentials of $\mathbf{B}$ and $\mathbf{B}_{\mathrm{p}}$, respectively, i.e., $\mathbf{B}=\nabla \times \mathbf{A}, \mathbf{B}_{\mathrm{p}}=\nabla \times \mathbf{A}_{\mathrm{p}}$. Here we compute the relative magnetic helicity following the method proposed by Valori et al. (2012). As shown in Figure 6(c), the relative helicity evolves very similar to that of the magnetic free energy, which is also consistent with the building up and strengthening of the MFR. The continuous injection of magnetic helicity and magnetic free energy should be attributed to the driving of photospheric magnetic field evolution.

\section{CONCLUSIONS}

Investigation of solar magnetic field configuration and evolution is essential for the understanding of the nature of solar eruptions. In this paper, we studied the formation process of an MFR that is associated with a major eruption event occurring on 2015 June 21 in AR 12371. We performed a data-driven MHD numerical simulation to recreate the $3 \mathrm{D}$ coronal magnetic evolution of this region. The simulation covers one-day time evolution before the eruption. Our model and analysis in details combining AIA observation from $S D O$ reveal the formation and evolution process of a long flux rope that is consistent with EUV observations but is not reconstructed from previous NLFFF extrapolations. We further computed the magnetic twist number, decay index, magnetic energy and helicity to investigate how the MFR originates, is built up and runs into an unstable state that is likely to trigger its eruption.

Our simulation has successfully and realistically generated the evolution of an MFR from the vector magnetogram. Compared with the previous work of Vemareddy \& Demóulin (2018) based on the static modeling through NLFFF method, the result of the selfconsistent MHD modelling offers a unique way to probe the dynamic formation process of the MFR. We can see the evolution of MFR before the eruption comprehensively and identify sophisticated structures like QSLs which may influence MFR's eruption.

Although it is now commonly believed that MFR plays a key role in solar eruptions, how and when an MFR associated with eruption forms is still under debates. Here we found that the MFR does not bodily emerge below the photosphere but forms gradually through magnetic reconnection in the corona before the flare. Comparison of a sequence of $S D O /$ AIA images with the reconstructed magnetic field topology confirms the existence of the MFR before the eruption in the analyzed event and shows the elongation of the MFR. From the simulation of the pre-flare magnetic topology, it is found that this long curved MFR was formed gradually above the PIL and extended out afterwards. Persistent injection of helicity and accumulation of magnetic free energy provide crucial ingredient for the building up of the MFR, which may result from the shear and rotation motion on the photosphere. Calculation of decay index suggests that the flux rope has reached an unstable region where TI may trigger the eruption.

In conclusion, all these findings demonstrate the complexity of pre-flare magnetic topology and disclose the formation and triggering mechanisms behind a largescale MFR. This study is important to understand the role of complex magnetic topology and also reveal the MFR formation progress before the flare in detail. More work is supposed to be done on this issue to determine fundamental triggering mechanisms of the eruption and better characterize the dynamics of solar eruptions.

The authors wish to thank the anonymous referee for his insightful comments in improving the paper. This work is supported by the National Natural Science Foundation of China (NSFC 41822404, 41731067, 41574170, 41531073), the Fundamental Research Funds for the Central Universities (Grant No.HIT.BRETIV.201901). 
Data from observations are courtesy of NASA SDO/AIA and the HMI science teams. The computation work was carried out on TianHe-1 (A) at the National Supercom- puter Center in Tianjin, China. C.W.J. thanks ISSI-BJ for supporting him to attending the team meeting led by J. C. Vial and P. F. Chen.

\section{REFERENCES}

Amari, T., Luciani, J. F., Aly, J. J., Mikic, Z., \& Linker, J. 2003, ApJ, 585, 1073

Archontis, V., Hood, A. W., Savcheva, A., Golub, L., \& Deluca, E. 2009, ApJ, 691, 1276

Aschwanden, M. J. 2004, Physics of the Solar Corona. An Introduction

Aulanier, G., Janvier, M., \& Schmieder, B. 2012, A\&A, 543, A110

Aulanier, G., Török, T., Démoulin, P., \& DeLuca, E. E. 2010, ApJ, 708, 314

Bateman, G. 1978, MHD instabilities

Benz, A. O. 2017, Living Reviews in Solar Physics, 14, 2

Berger, M. A., \& Field, G. B. 1984, Journal of Fluid Mechanics, 147, 133

Berger, M. A., \& Prior, C. 2006, Journal of Physics A Mathematical General, 39, 8321

Bobra, M. G., Sun, X., Hoeksema, J. T., et al. 2014, SoPh, 289, 3549

Carmichael, H. 1964, NASA Special Publication, 50, 451

Chen, P. F. 2011, Living Reviews in Solar Physics, 8, 1

Cheng, X., Guo, Y., \& Ding, M. 2017, Science in China Earth Sciences, 60, 1383

Cheng, X., Zhang, J., Ding, M. D., Liu, Y., \& Poomvises, W. 2013, ApJ, 763, 43

Cheng, X., Zhang, J., Saar, S. H., \& Ding, M. D. 2012, ApJ, 761, 62

Cheng, X., Ding, M. D., Guo, Y., et al. 2014, ApJ, 780, 28

Cheung, M., Rempel, M., Chintzoglou, G., et al. 2019, Nature Astronomy, 3, 160

Cheung, M. C. M., \& DeRosa, M. L. 2012, ApJ, 757, 147

Démoulin, P. 2006, Advances in Space Research, 37, 1269

Duan, A., Jiang, C., He, W., et al. 2019, The Astrophysical Journal, 884, 73

Fan, Y. 2001, ApJ, 554, L111

Fan, Y., \& Gibson, S. E. 2007, ApJ, 668, 1232

Finn, J. 1984, Comments Plasma Phys. Controlled Fusion, 9, 111

Forbes, T. G. 2000, Journal of Geophysical Research, 105, 23153

Gibson, S. E., \& Fan, Y. 2006, ApJL, 637, L65

Gou, T., Liu, R., Kliem, B., Wang, Y., \& Veronig, A. M. 2019, Science Advances, 5, 7004

Guo, Y., Xia, C., Keppens, R., Ding, M. D., \& Chen, P. F. 2019, The Astrophysical Journal, 870, L21

Hayashi, K., Feng, X., Xiong, M., \& Jiang, C. 2019, The Astrophysical Journal, 871, L28

Hirayama, T. 1974, SoPh, 34, 323

Jiang, C., \& Feng, X. 2013, ApJ, 769, 144
-. 2014, SoPh, 289, 63

Jiang, C., Feng, X., \& Hu, Q. 2018a, ApJ, 866, 96

Jiang, C., Feng, X., Wu, S. T., \& Hu, Q. 2013, ApJL, 771, L30

Jiang, C., Feng, X., Zhang, J., \& Zhong, D. 2010, SoPh, 267, 463

Jiang, C., Wu, S. T., Feng, X., \& Hu, Q. 2016, Nature Communications, 7, 11522

Jiang, C., Yan, X., Feng, X., et al. 2017, ApJ, 850, 8

Jiang, C., Zou, P., Feng, X., et al. 2018b, ApJ, 869, 13

Kliem, B., \& Török, T. 2006, PhRvL, 96, 255002

Kopp, R. A., \& Pneuman, G. W. 1976, SoPh, 50, 85

Lemen, J. R., Title, A. M., Akin, D. J., et al. 2012, SoPh, 275, 17

Liu, R., Kliem, B., Titov, V. S., et al. 2016, ApJ, 818, 148

Low, B. C., \& Lou, Y. Q. 1990, ApJ, 352, 343

Magara, T. 2004, ApJ, 605, 480

Martínez-Sykora, J., Hansteen, V., \& Carlsson, M. 2008, ApJ, 679,871

Moore, R. L., Sterling, A. C., Hudson, H. S., \& Lemen, J. R. 2001, ApJ, 552, 833

Myers, C. E., Yamada, M., Ji, H., et al. 2015, Nature, 528, 526

Nayak, S. S., Bhattacharyya, R., Prasad, A., et al. 2019, ApJ, 875,10

Ouyang, Y., Zhou, Y. H., Chen, P. F., \& Fang, C. 2017, The Astrophysical Journal, 835, 94

Pomoell, J., Lumme, E., \& Kilpua, E. 2019, SoPh, 294, 41

Priest, E. R., \& Démoulin, P. 1995, Journal of Geophysical Research, 100, 23443

Priest, E. R., \& Forbes, T. G. 2002, Astronomy and Astrophysics Review, 10, 313

Savcheva, A., Pariat, E., van Ballegooijen, A., Aulanier, G., \& DeLuca, E. 2012, The Astrophysical Journal, 750, 15

Savcheva, A. S., van Ballegooijen, A. A., \& DeLuca, E. E. 2012, ApJ, 744, 78

Schou, J., Scherrer, P. H., Bush, R. I., et al. 2012, SoPh, 275, 229

Schuck, P. W. 2008, The Astrophysical Journal, 683, 1134

Sturrock, P. A. 1966, Nature, 211, 695

Titov, V. S., \& Démoulin, P. 1999, A\&A, 351, 707

Valori, G., Démoulin, P., \& Pariat, E. 2012, SoPh, 278, 347

van Ballegooijen, A. A., \& Martens, P. C. H. 1989, ApJ, 343, 971

Vemareddy, P. 2017, ApJ, 845, 59

Vemareddy, P., \& Demóulin, P. 2018, ApJ, 857, 90

Wu, S. T., Wang, A. H., Liu, Y., \& Hoeksema, J. T. 2006, ApJ, 652,800

Zhang, J., Cheng, X., \& Ding, M.-D. 2012, Nature Communications, 3, 747 